\title{
Brief Report \\ Inhibition of LpxC Increases the Activity of Iron Chelators and Gallium Nitrate in Multidrug-Resistant Acinetobacter baumannii
}

\author{
Víctor Vinuesa ${ }^{1}{ }^{1}$, Raquel Cruces ${ }^{1}$, Francesca Nonnoi ${ }^{1}$ and Michael J. McConnell ${ }^{2, *}$ \\ 1 Intrahospital Infections Laboratory, National Centre for Microbiology, Instituto de Salud Carlos III, \\ 28220 Madrid, Spain; vvinuesa@isciii.es (V.V.); rcrufer@gmail.com (R.C.); francesca.nonnoi@gmail.com (F.N.) \\ 2 Vaxdyn S.L., Avenida Manuel Siurot s/n., 41010 Seville, Spain \\ * Correspondence: michael.mcconnell@isciii.es; Tel.: +34-918-223-869
}

check for updates

Citation: Vinuesa, V.; Cruces, R.; Nonnoi, F.; McConnell, M.J. Inhibition of LpxC Increases the Activity of Iron Chelators and Gallium Nitrate in Multidrug-Resistant Acinetobacter baumannii. Antibiotics 2021, 10, 609. https://doi.org/10.3390/ antibiotics 10050609

Academic Editor: Carlos M. Franco

Received: 16 April 2021

Accepted: 18 May 2021

Published: 20 May 2021

Publisher's Note: MDPI stays neutral with regard to jurisdictional claims in published maps and institutional affiliations.

Copyright: (c) 2021 by the authors. Licensee MDPI, Basel, Switzerland. This article is an open access article distributed under the terms and conditions of the Creative Commons Attribution (CC BY) license (https:// creativecommons.org/licenses/by/ $4.0 /)$.

\begin{abstract}
Infections caused by multidrug-resistant Acinetobacter baumannii would benefit from the development of novel treatment approaches. Compounds that interfere with bacterial iron metabolism, such as iron chelators and gallium nitrate, have previously been shown to have antimicrobial activity against $A$. baumannii. In this study, we characterize the effect of LpxC inhibitors on the antimicrobial activity of previously characterized iron chelators, 2,2'-bipyridyl (BIP) and deferiprone (DFP), and gallium nitrate $\left(\mathrm{Ga}\left(\mathrm{NO}_{3}\right)_{3}\right)$ against $A$. baumannii reference strains and multidrug-resistant clinical isolates. The LpxC inhibitor LpxC-2 was synergistic with BIP for $30 \%$ of strains tested (FICI values: 0.38-1.02), whereas inhibition with LpxC-4 was synergistic with BIP for $60 \%$ of strains tested (FICI values: 0.09-0.75). In time-kill assays, combinations of BIP with both LpxC inhibitors demonstrated synergistic activity, with a more than $3 \log _{10}$ reduction in bacterial counts compared to BIP alone. LpxC-2 was synergistic with $\mathrm{Ga}\left(\mathrm{NO}_{3}\right)_{3}$ for $50 \%$ of strains tested (FICI values: $0.27-1.0$ ), whereas LpxC-4 was synergistic with $\mathrm{Ga}\left(\mathrm{NO}_{3}\right)_{3}$ for all strains tested (FICI values: $\left.0.08-\leq 0.50\right)$. In time-kill assays, combinations of $\mathrm{Ga}\left(\mathrm{NO}_{3}\right)_{3}$ with $\mathrm{LpxC}-2$ and $\mathrm{LpxC}-4$ decreased the growth of both strains compared to each compound separately; however, only the combination with LpxC-4 met the defined criteria for synergy. These results identify a novel synergy between two antimicrobial classes against A. baumannii strains.
\end{abstract}

Keywords: LpxC inhibitors; iron chelators; gallium; antibiotic resistance; synergy

\section{Introduction}

The emergence and dissemination of infections caused by multidrug-resistant Acinetobacter baumannii warrants the development of new treatment and prevention strategies [1]. The identification of compounds that can increase the activity of existing antimicrobials and the characterization of combinations of compounds that demonstrate synergistic activity against multidrug-resistant $A$. baumannii may represent a promising approach. Inhibitors of LpxC, a zinc-dependent deacetylase that catalyzes one of the initial reactions in the biosynthesis of lipid A, are currently being developed as antimicrobial agents for the treatment of infections caused by Gram-negative pathogens [2]. LpxC inhibitors demonstrate potent activity against multiple species associated with multidrug resistance, including Pseudomonas aeruginosa, Escherichia coli and Klebsiella pneumoniae. However, these compounds are generally less active against $A$. baumannii isolates, in spite of the fact that $\mathrm{LpxC}$ is required for lipid A biosynthesis in $A$. baumannii and that the enzymatic activity of $A$. baumannii LpxC is inhibited by LpxC inhibitors, which demonstrate activity against other Gram-negative species [3-5]. This difference is likely due to the viability of $A$. baumannii in the absence of lipid A/lipooligosaccharide (LOS) biosynthesis, as indicated by multiple studies demonstrating the growth and survival of $A$. baumannii strains completely deficient in lipid A due to mutations in the $\operatorname{lp} x A, \operatorname{lp} x C$ and $\operatorname{lp} x D$ genes [6-8]. This is in contrast to the majority of Gram-negative species, which require lipid A biosynthesis for viability [9]. 
Iron is required for multiple bacterial processes and is thus essential for establishing host infection. For this reason, iron chelators have been explored as potential antimicrobial agents for multiple bacterial species in both in vitro and in vivo studies [10]. Multiple iron-chelating compounds have demonstrated antimicrobial activity against $A$. baumannii, including 2,2'-bipyridyl (BIP), deferiprone (DFP) and DIBI, among others (reviewed in [10]). In addition, gallium, an iron mimetic that interferes with bacterial iron metabolism, has demonstrated activity against different $A$. baumannii strains [11-13], and has been evaluated as an antimicrobial agent in a clinical trial [14].

In a previous study, we showed that $A$. baumannii strains deficient in lipid A biosynthesis due to mutations in $l p x A, l p x C$ and $l p x D$ genes demonstrate decreased growth, compared to wild-type parental strains in the presence of the iron chelator BIP [8]. Based on these findings, we hypothesize that pharmacologic inhibition of lipid A biosynthesis using LpxC inhibitors may increase the susceptibility of $A$. baumannii to iron chelators and galliumcontaining compounds. In this study, we characterize the effect of LpxC inhibition on the antimicrobial activity of previously characterized iron chelators, 2,2'-bipyridyl (BIP) and deferiprone (DFP), and gallium nitrate $\left(\mathrm{Ga}\left(\mathrm{NO}_{3}\right)_{3}\right.$ against $A$. baumannii reference strains and multidrug-resistant clinical isolates.

\section{Results}

\subsection{Checkerboard Assays with A. baumannii Reference Strains}

In order to initially characterize potential synergistic activity between LpxC inhibitors and iron-chelating or gallium compounds, the A. baumannii reference strains ATCC 179878 and ATCC 19606 were employed in checkerboard assays with the iron chelators BIP, DFP and $\mathrm{Ga}\left(\mathrm{NO}_{3}\right)_{3}$, and the previously described LpxC inhibitors PF-4753299 (LpxC-2) and PF-5081090 (LpxC-4) [5]. Importantly, checkerboard assays were performed in RPMI 1640 , as previously described [15], in order to perform assays under conditions that more closely mimic iron concentrations encountered by A. baumannii during infection. Minimal inhibitory concentrations for ATCC 19606 and ATCC 17978 for the compounds used in this study are shown in Table 1.

Table 1. MICs of iron chelators, gallium nitrate and LpxC inhibitors for a A. baumannii strains used in this study.

\begin{tabular}{cccccc}
\hline \multirow{2}{*}{ Strain } & BIP & DFP & Ga(NO $\mathbf{N}_{\mathbf{3}} \mathbf{3}$ & LpxC-2 & LpxC-4 \\
\cline { 2 - 6 } & 64 & 128 & 128 & 16 & 512 \\
\hline ATCC 17978 & 64 & 128 & 128 & 32 & 512 \\
ATCC 19606 & 64 & ND & 128 & 16 & $>512$ \\
$\mathrm{Ab}-1$ & 64 & ND & 128 & 32 & $>512$ \\
$\mathrm{Ab}-66$ & 64 & ND & 128 & 16 & $>512$ \\
$\mathrm{Ab}-84$ & 64 & ND & 32 & 16 & $>512$ \\
$\mathrm{Ab}-108$ & 64 & ND & 64 & 16 & 512 \\
$\mathrm{Ab}-154$ & 32 & ND & 128 & 128 & $>512$ \\
$\mathrm{Ab}-167$ & 64 & ND & 64 & 16 & $>512$ \\
$\mathrm{Ab}-176$ & 64 & ND & 128 & 8 & $>512$ \\
$\mathrm{Ab}-208$ & 64 & &
\end{tabular}

BIP, 2,2'-bipyridyl; DFP, deferiprone; $\mathrm{Ga}(\mathrm{NO} 3) 3$, gallium nitrate; ND, not determined.

The fractional inhibitory concentration index (FICI) was calculated for each combination, and synergy was defined as a FICI $\leq 0.5$, indifference as a FICI of $>0.5$ but $\leq 4$ and antagonism as FICI $>4$, as previously described [15]. As can be seen in Table 2, DFP did not demonstrate synergistic activity with either of the LpxC inhibitors. Synergistic activity with combinations of BIP and the LpxC inhibitors was strain-dependent, with LpxC-2 and BIP demonstrating synergy against the ATCC 19606 strain, and LpxC-4 and BIP demonstrating synergy against ATCC 17978. Interestingly, combinations of LpxC-2 or LpxC-4 with $\mathrm{Ga}\left(\mathrm{NO}_{3}\right)_{3}$ were highly synergistic for both reference strains (FICI values 0.08-0.38). 
Table 2. Checkerboard assay for combinations of BIP, DFP and $\mathrm{Ga}\left(\mathrm{NO}_{3}\right)_{3}$ and $\mathrm{LpxC}$ inhibitors with a panel of

A. baumannii strains.

\begin{tabular}{|c|c|c|c|c|c|c|c|c|c|c|c|c|}
\hline \multirow{3}{*}{ Strain } & \multicolumn{4}{|c|}{ BIP } & \multicolumn{4}{|c|}{ DFP } & \multicolumn{4}{|c|}{$\mathrm{Ga}\left(\mathrm{NO}_{3}\right)_{3}$} \\
\hline & \multicolumn{2}{|c|}{ LpxC-2 } & \multicolumn{2}{|r|}{ LpxC-4 } & \multicolumn{2}{|r|}{ LpxC-2 } & \multicolumn{2}{|r|}{ LpxC-4 } & \multicolumn{2}{|r|}{ LpxC-2 } & \multicolumn{2}{|r|}{ LpxC-4 } \\
\hline & $\begin{array}{l}\text { FICI } \\
\text { Value }\end{array}$ & $\begin{array}{c}\text { Effect of } \\
\text { Combination }\end{array}$ & $\begin{array}{l}\text { FICI } \\
\text { Value }\end{array}$ & $\begin{array}{c}\text { Effect of } \\
\text { Combination }\end{array}$ & $\begin{array}{c}\text { FICI } \\
\text { Value }\end{array}$ & $\begin{array}{c}\text { Effect of } \\
\text { Combination }\end{array}$ & $\begin{array}{c}\text { FICI } \\
\text { Value }\end{array}$ & $\begin{array}{c}\text { Effect of } \\
\text { Combination }\end{array}$ & $\begin{array}{l}\text { FICI } \\
\text { Value }\end{array}$ & $\begin{array}{c}\text { Effect of } \\
\text { Combination }\end{array}$ & $\begin{array}{c}\text { FICI } \\
\text { Value }\end{array}$ & $\begin{array}{c}\text { Effect of } \\
\text { Combination }\end{array}$ \\
\hline $\begin{array}{l}\text { ATCC } \\
17978\end{array}$ & 0.53 & Indifference & 0.09 & Synergy & 1.01 & Indifference & 1.00 & Indifference & 0.38 & Synergy & 0.38 & Synergy \\
\hline $\begin{array}{l}\text { ATCC } \\
19606\end{array}$ & 0.50 & Synergy & 0.53 & Indifference & 1.00 & Indifference & 1.00 & Indifference & 0.27 & Synergy & 0.08 & Synergy \\
\hline Ab-66 & 0.38 & Synergy & $\leq 0.50$ & Synergy & ND & - & ND & - & 0.38 & Synergy & $\leq 0.19$ & Synergy \\
\hline $\mathrm{Ab}-84$ & 1.00 & Indifference & $\leq 0.63$ & Indifference & ND & - & ND & - & 0.50 & Synergy & $\leq 0.09$ & Synergy \\
\hline Ab-108 & 0.63 & Indifference & $\leq 0.50$ & Synergy & ND & - & ND & - & 0.75 & Indifference & $\leq 0.16$ & Synergy \\
\hline Ab-154 & 0.56 & Indifference & 0.75 & Indifference & ND & - & ND & - & 0.53 & Indifference & 0.31 & Synergy \\
\hline Ab-167 & 0.50 & Synergy & $\leq 0.75$ & Indifference & ND & - & ND & - & 0.53 & Indifference & $\leq 0.50$ & Synergy \\
\hline Ab-176 & 0.52 & Indifference & $\leq 0.38$ & Synergy & ND & - & ND & - & 0.50 & Synergy & $\leq 0.19$ & Synergy \\
\hline$A b-208$ & 0.53 & Indifference & $\leq 0.38$ & Synergy & ND & - & ND & - & 0.75 & Indifference & $\leq 0.09$ & Synergy \\
\hline
\end{tabular}

BIP, 2,2'-bipyridyl; DFP, deferiprone; FICI, fractional inhibitory concentration index; $\mathrm{Ga}\left(\mathrm{NO}_{3}\right)_{3}$, gallium nitrate; $\mathrm{ND}$, not determined. 


\subsection{Time-Kill Analysis of Combinations of LpxC Inhibitors and Iron Chelators with A. baumannii Reference Strains}

In order to further characterize the antimicrobial activity of combinations of LpxC inhibitors and iron chelators, time-kill studies were performed in RPMI medium. BIP and DFP were used at the MIC value and LpxC inhibitors were used at a concentration of $1 / 4$ the MIC. Synergy was defined as a $2 \log _{10}$ decrease in colony counts at $24 \mathrm{~h}$ with the combination of compounds compared to the most active single agent alone. As shown in Figure 1A,B, combinations of BIP with both LpxC inhibitors demonstrated synergistic activity, with a $\sim 4 \log _{10}$ reduction in bacterial counts compared to BIP alone in the case of the ATCC 17978 strain, and more than $3 \log _{10}$ for the ATCC 19606 strain. The combinations of LpxC-2 and LpxC-4 with DFP reduced the growth of both strains with respect to the most active single compound, although this reduction was never greater than $1 \log _{10}$ after $24 \mathrm{~h}$ of incubation (Figure 1C,D).

\subsection{Time-Kill Analysis of Combinations of LpxC Inhibitors and $\mathrm{Ga}\left(\mathrm{NO}_{3}\right)_{3}$ with A. baumannii Reference Strains}

Time-kill studies were performed in RPMI medium with $\mathrm{Ga}\left(\mathrm{NO}_{3}\right)_{3}$ at the MIC value and LpxC inhibitors at a concentration of $1 / 4$ the MIC, as described in the previous section. Combinations of $\mathrm{Ga}\left(\mathrm{NO}_{3}\right)_{3}$ with LpxC-2 and LpxC-4 decreased the growth of both strains compared to each compound separately; however, only the combination with LpxC-4 met the defined criteria for synergy (Figure 2A,B). In addition to the testing combinations of $\mathrm{Ga}\left(\mathrm{NO}_{3}\right)_{3}$ at the MIC (128 mg/L), synergistic activity of this compound with the LpxC inhibitors at $32 \mathrm{mg} / \mathrm{L}$ was determined since this concentration has previously been reported to be nontoxic in humans [16]. $\mathrm{Ga}\left(\mathrm{NO}_{3}\right)_{3}$ at this concentration, combined with LpxC-2 at 1/4x MIC, had no greater effect than the most active compound alone (Figure 2C,D), whereas this concentration in combination with LpxC-4 at $1 / 4 \times$ MIC reduced bacterial counts with respect to the most active single compound for both ATCC 19606 and ATCC 17978 strains $\left(1.1 \log _{10}\right.$ and $3.6 \log _{10}$, respectively). These results indicate that therapeutic concentrations of $\mathrm{Ga}\left(\mathrm{NO}_{3}\right)_{3}$ can act synergistically with subinhibitory concentrations of LpxC-4.

A

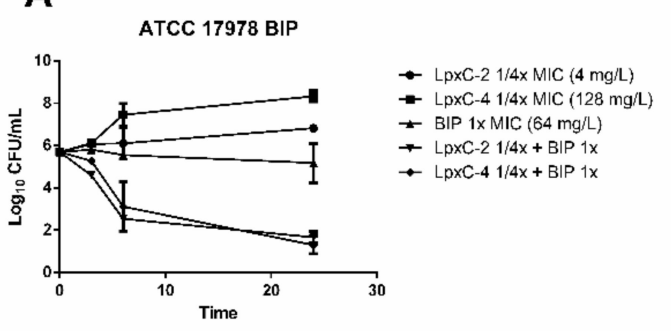

C

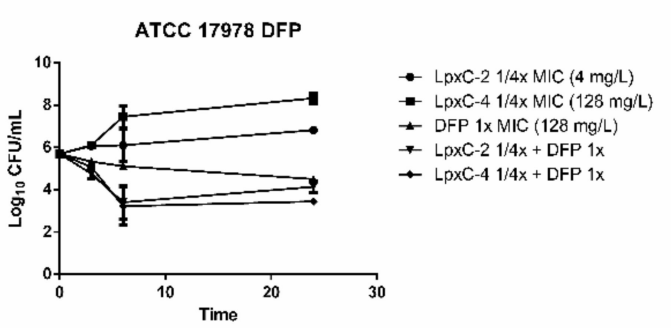

B

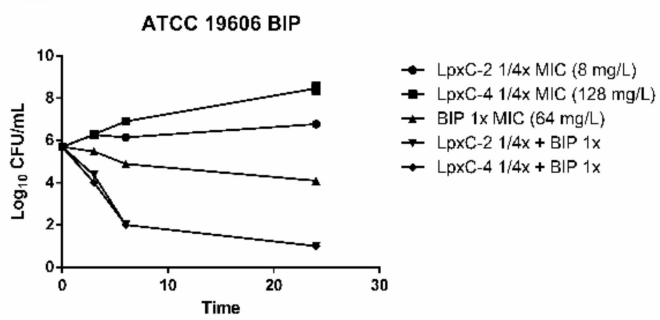

D

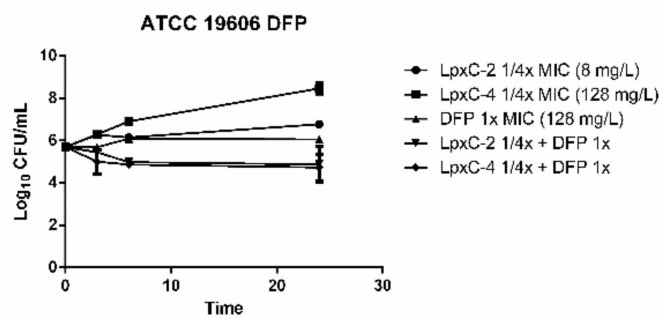

Figure 1. Time-kill curves for iron chelators in combination with LpxC inhibitors. Time-kill curves indicating bacterial counts in cultures at $0,3,6$ and $24 \mathrm{~h}$ with the indicated A. baumannii strains containing combinations of BIP with LpxC-2 and LpxC-4 (A,B) or DFP with LpxC-2 and LpxC-4 (C,D). The indicated concentrations refer to the final concentration of each compound in the bacterial culture. BIP, 2,2'-bipyridyl; DFP, deferiprone. Time points indicate the mean bacterial count from a minimum of two biological replicates and error bars represent the standard deviation. 


\subsection{Activity of LpxC Inhibitors in Combination with Iron Chelators and Gallium Nitrate in A. baumannii Clinical Isolates}

In order to determine if combinations of LpxC inhibitors and BIP or $\mathrm{Ga}\left(\mathrm{NO}_{3}\right)_{3}$ have synergistic activity against $A$. baumannii clinical isolates, checkerboard assays were carried out as described above with eight previously characterized clinical isolates $[17,18]$. DFP was not included in this analysis since it did not demonstrate synergy in either checkerboard or time-kill assays with the ATCC 17978 and ATCC 19606 reference strains. The activity of BIP in combination with LpxC-2 and LpxC-4 was strain-dependent, as the combination $\mathrm{BIP} / \mathrm{LpxC}-2$ was synergistic in 2 of 8 strains tested, and the BIP/LpxC-4 demonstrated synergy in 5 of 8 strains (Table 2). The synergistic activity of $\mathrm{Ga}\left(\mathrm{NO}_{3}\right)_{3}$ in combination with LpxC-2 was also strain-dependent, demonstrating synergy in 3 of 8 clinical isolates. Interestingly, $\mathrm{Ga}\left(\mathrm{NO}_{3}\right)_{3}$ in combination with LpxC-4 was highly synergistic against all clinical isolates, with FICI values between $\leq 0.09$ and 0.31 . These results indicate that some combinations of LpxC inhibitors with iron chelators or gallium demonstrate synergy in a strain-dependent manner, whereas the combination of LpxC-4 and $\mathrm{Ga}\left(\mathrm{NO}_{3}\right)_{3}$ was synergistic in all strains.

A

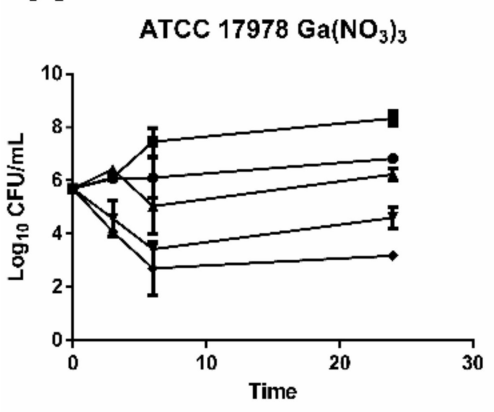

- LpxC-2 1/4x MIC (4 mg/L)

- LpxC-4 1/4x MIC (128 mg/L)

- $\mathrm{Ga}\left(\mathrm{NO}_{3}\right)_{3} 1 \times \mathrm{MIC}(128 \mathrm{mg} / \mathrm{L})$

$\rightarrow \mathrm{LpxC}-21 / 4 \mathrm{x}+\mathrm{Ga}\left(\mathrm{NO}_{3}\right)_{3} 1 \mathrm{x}$

$\rightarrow \mathrm{LpxC}-41 / 4 \mathrm{x}+\mathrm{Ga}\left(\mathrm{NO}_{3}\right)_{3} 1 \mathrm{x}$
B

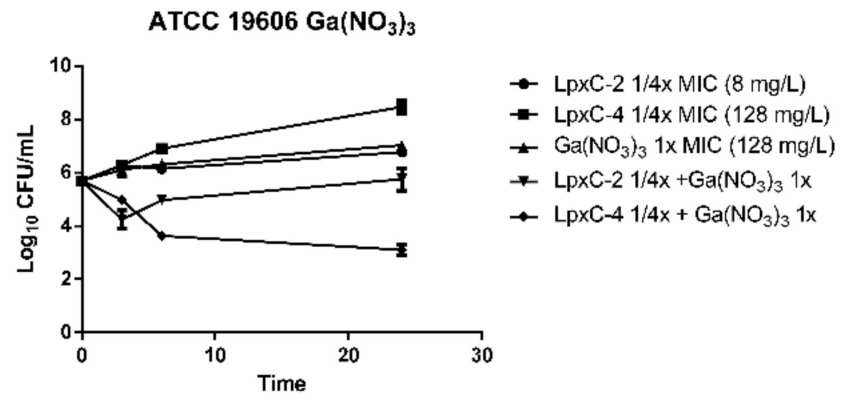

D

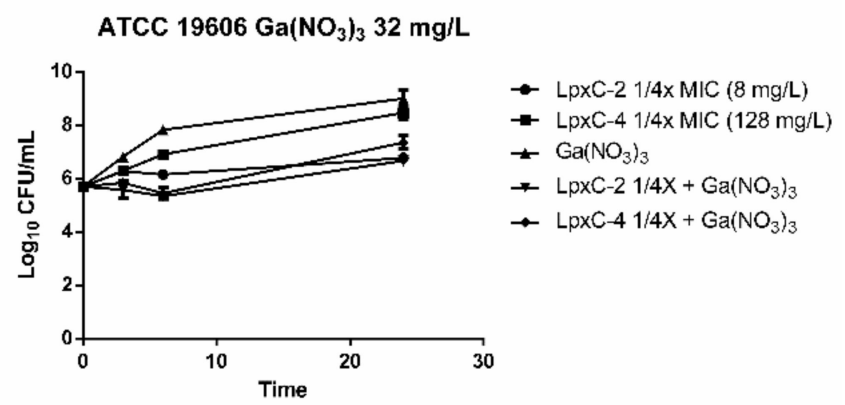

Figure 2. Time-kill curves for gallium nitrate in combination with LpxC inhibitors. Time-kill curves indicating bacterial counts in cultures at $0,3,6$ and $24 \mathrm{~h}$ with the indicated $A$. baumannii strains containing combinations of $\mathrm{Ga}\left(\mathrm{NO}_{3}\right)_{3}$ with LpxC-2 and LpxC-4 (A-D) at the indicated concentrations. Time points indicate the mean bacterial count from a minimum of two biological replicates and error bars represent the standard deviation. $\mathrm{Ga}\left(\mathrm{NO}_{3}\right)_{3}$, gallium nitrate.

\section{Discussion}

The results of this study characterize novel synergistic combinations of LpxC inhibitors and compounds that interfere with bacterial iron metabolism, although the mechanisms underlying this synergistic activity are unclear. A previous study demonstrated that inhibition of LpxC in A. baumannii increases cell permeability and susceptibility to azithromycin, vancomycin and rifampin [19]. In this previous study, it was hypothesized that the increased cell permeability may contribute to the increased activity of these antimicrobials. 
Given that both BIP and $\mathrm{Ga}\left(\mathrm{NO}_{3}\right)_{3}$ have been proposed to act intracellularly $[16,20]$, one possibility is that increased cell permeability due to LpxC inhibition permits greater intracellular accumulation of these compounds in A. baumannii. In contrast, the antimicrobial activity of deferiprone is thought to be primarily due to its iron-chelating activity in the extracellular space, which may explain why LpxC inhibition was less effective at increasing the activity of this compound.

LpxC inhibitors, iron chelators and gallium compounds are currently being developed as therapeutics for multidrug-resistant Gram-negative infections $[2,10]$. The results of this study indicate that combinations of these compounds may be synergistic against multidrugresistant $A$. baumannii and may thus warrant further study. The combination of LpxC-4 together with gallium nitrate may be of particular interest given that it was highly synergistic against all strains tested. This study thus lays the groundwork for exploring synergistic combinations of LpxC inhibitors and compounds that interfere with iron metabolism, and may therefore have application in the clinical management of multidrug-resistant infections caused by A. baumannii.

\section{Materials and Methods}

\subsection{A. baumannii Strains Used in This Study}

The antibiotic-susceptible A. baumannii reference strains ATCC 19606 and ATCC 17978 were used. In addition, 8 previously characterized $A$. baumannii clinical isolates were employed in checkerboard assays $[17,18]$. All strains were maintained on LB agar and growth in LB broth before checkerboard and time-kill assays, unless otherwise stated.

\subsection{LpxC Inhibitors, Iron Chelators and Gallium Nitrate}

BIP, DFP and $\mathrm{Ga}\left(\mathrm{NO}_{3}\right)_{3}$ were purchased from Sigma and stock solutions were dissolved in water for DFP and $\mathrm{Ga}\left(\mathrm{NO}_{3}\right)_{3}$ and ethanol for BIP. The previously described LpxC inhibitors, PF-4753299 (LpxC-2) and PF-5081090 (LpxC-4) [5], were purchased from Axon Medchem (Groningen, Netherlands) and dissolved in DMSO. All reagents were prepared as $1024 \mathrm{mg} / \mathrm{L}$ stock solutions and stored at $-20^{\circ} \mathrm{C}$ until use.

\subsection{Checkerboard Assays}

Checkerboard assays were performed in RPMI 1640 as previously described [15], with modification. Briefly, bacterial cultures grown overnight on LB agar were re-suspended in RPMI, adjusted to an $\mathrm{OD}_{600}$ of $0.05-0.15$ and diluted 1:10 in RPMI to yield a suspension of $1 \times 10^{6} \mathrm{CFU} / \mathrm{mL}$. Fifty $\mu \mathrm{L}$ of this suspension was combined with $50 \mu \mathrm{L}$ of 7 two-fold dilutions of BIP, DFP or $\mathrm{Ga}\left(\mathrm{NO}_{3}\right)_{3}$ in combination with 9 two-fold dilutions of LpxC-2 or LpxC-4 and incubated at $37^{\circ} \mathrm{C}$ for $24 \mathrm{~h}$. The starting concentration for each compound was $256 \mathrm{mg} / \mathrm{L}$. Growth inhibition was determined by visual inspection of turbidity in assay wells. All strains were assayed with at least two biological replicates.

\subsection{Time-Kill Analysis}

Time-kill assays were carried out in RPMI 1640 media with bacterial suspension at an initial concentration of $5 \times 10^{5} \mathrm{CFU} / \mathrm{mL}$ in a final volume of $2 \mathrm{~mL}$. BIP, DFP and $\mathrm{Ga}\left(\mathrm{NO}_{3}\right)_{3}$ were used at the MIC value and LpxC inhibitors were used at a concentration of $1 / 4$ the MIC, unless otherwise indicated. Cultures were continuously shaken at $37^{\circ} \mathrm{C}$ and samples were taken at 0, 3, 6 and $24 \mathrm{~h}$ for bacterial quantification on LB agar. Synergy was defined as a $2 \log _{10}$ decrease in colony counts at $24 \mathrm{~h}$ with the combination of compounds compared to the most active single agent alone. All strains were assayed with at least two biological replicates.

Author Contributions: Conceptualization, V.V. and M.J.M.; methodology, V.V. and M.J.M.; formal analysis, V.V. and M.J.M.; investigation, V.V., R.C., F.N. and M.J.M.; resources, M.J.M.; data curation, M.J.M.; writing—original draft preparation, V.V. and M.J.M.; writing—review and editing, V.V. and M.J.M.; funding acquisition, V.V. and M.J.M. All authors have read and agreed to the published version of the manuscript. 
Funding: This research was supported by grants MPY 380/18 from the Instituto de Salud Carlos III (ISCIII) awarded to M.J.M. V.V. is supported by the Río Hortega Program from the Instituto de Salud Carlos III. The APC was funded by MPY 380/18 from the Instituto de Salud Carlos III (ISCIII) awarded to M.J.M.

Institutional Review Board Statement: Not applicable.

Informed Consent Statement: Not applicable.

Data Availability Statement: Data is available from the authors upon request.

Acknowledgments: This research was supported by grants MPY 380/18 from the Instituto de Salud Carlos III (ISCIII) awarded to M.J.M. V.V. is supported by the Río Hortega Program from the Instituto de Salud Carlos III.

Conflicts of Interest: M.J.M. is a founder and shareholder in the biotechnology company Vaxdyn, S.L. Vaxdyn played no role in the present study. No other competing interest is declared.

\section{References}

1. McConnell, M.J.; Actis, L.; Pachón, J. Acinetobacter baumannii: Human infections, factors contributing to pathogenesis and animal models. FEMS Microbiol. Rev. 2013, 37, 130-155. [CrossRef] [PubMed]

2. Liu, F.; Ma, S. Recent Process in the Inhibitors of UDP-3-O-(R-3-hydroxyacyl)-Nacetylglucosamine Deacetylase (LpxC) Against Gram-Negative Bacteria. Mini Rev. Med. Chem. 2018, 18, 310-323. [CrossRef] [PubMed]

3. Lin, L.; Tan, B.; Pantapalangkoor, P.; Ho, T.; Baquir, B.; Tomaras, A.; Montgomery, J.I.; Reilly, U.; Barbacci, E.G.; Hujer, K.; et al. Inhibition of LpxC protects mice from resistant Acinetobacter baumannii by modulating inflammation and enhancing phagocytosis. mBio 2012, 3. [CrossRef] [PubMed]

4. Montgomery, J.I.; Brown, M.F.; Reilly, U.; Price, L.M.; Abramite, J.A.; Arcari, J.; Barham, R.; Che, Y.; Chen, J.M.; Chung, S.W.; et al. Pyridone methylsulfone hydroxamate LpxC inhibitors for the treatment of serious gram-negative infections. J. Med. Chem. 2012, 55, 1662-1670. [CrossRef] [PubMed]

5. Tomaras, A.P.; McPherson, C.J.; Kuhn, M.; Carifa, A.; Mullins, L.; George, D.; Desbonnet, C.; Eidem, T.M.; Montgomery, J.I.; Brown, M.F.; et al. LpxC inhibitors as new antibacterial agents and tools for studying regulation of lipid A biosynthesis in Gram-negative pathogens. mBio 2014, 5, e01551-14. [CrossRef] [PubMed]

6. Moffatt, J.H.; Harper, M.; Harrison, P.; Hale, J.D.; Vinogradov, E.; Seemann, T.; Henry, R.; Crane, B.; St Michael, F.; Cox, A.D.; et al. Colistin resistance in Acinetobacter baumannii is mediated by complete loss of lipopolysaccharide production. Antimicrob. Agents Chemother. 2010, 54, 4971-4977. [CrossRef] [PubMed]

7. Moffatt, J.H.; Harper, M.; Adler, B.; Nation, R.L.; Li, J.; Boyce, J.D. Insertion sequence ISAba11 is involved in colistin resistance and loss of lipopolysaccharide in Acinetobacter baumannii. Antimicrob. Agents Chemother. 2011, 55, 3022-3024. [CrossRef] [PubMed]

8. Carretero-Ledesma, M.; García-Quintanilla, M.; Martín-Peña, R.; Pulido, M.R.; Pachón, J.; McConnell, M.J. Phenotypic changes associated with Colistin resistance due to Lipopolysaccharide loss in Acinetobacter baumannii. Virulence 2018, 9, 930-942. [CrossRef]

9. Zhang, G.; Meredith, T.C.; Kahne, D. On the essentiality of lipopolysaccharide to Gram-negative bacteria. Curr. Opin. Microbiol. 2013, 16, 779-785. [CrossRef] [PubMed]

10. Vinuesa, V.; McConnell, M.J. Recent Advances in Iron Chelation and Gallium-Based Therapies for Antibiotic Resistant Bacterial Infections. Int. J. Mol. Sci. 2021, 22, 2876. [CrossRef]

11. Antunes, L.C.; Imperi, F.; Minandri, F.; Visca, P. In vitro and in vivo antimicrobial activities of gallium nitrate against multidrugresistant Acinetobacter baumannii. Antimicrob. Agents Chemother. 2012, 56, 5961-5970. [CrossRef] [PubMed]

12. Choi, S.R.; Britigan, B.E.; Narayanasamy, P. Dual Inhibition of Klebsiella pneumoniae and Pseudomonas aeruginosa Iron Metabolism Using Gallium Porphyrin and Gallium Nitrate. ACS Infect. Dis. 2019, 5, 1559-1569. [CrossRef] [PubMed]

13. Hijazi, S.; Visaggio, D.; Pirolo, M.; Frangipani, E.; Bernstein, L.; Visca, P. Antimicrobial Activity of Gallium Compounds on ESKAPE Pathogens. Front. Cell Infect. Microbiol. 2018, 8, 316. [CrossRef] [PubMed]

14. Goss, C.H.; Kaneko, Y.; Khuu, L.; Anderson, G.D.; Ravishankar, S.; Aitken, M.L.; Lechtzin, N.; Zhou, G.; Czyz, D.M.; McLean, K.; et al. Gallium disrupts bacterial iron metabolism and has therapeutic effects in mice and humans with lung infections. Sci. Transl. Med. 2018, 10, eaat7520. [CrossRef] [PubMed]

15. Petersen, P.J.; Labthavikul, P.; Jones, C.H.; Bradford, P.A. In vitro antibacterial activities of tigecycline in combination with other antimicrobial agents determined by chequerboard and time-kill kinetic analysis. J. Antimicrob. Chemother. 2006, 57, 573-576. [CrossRef] [PubMed]

16. Bernstein, L.R. Mechanisms of therapeutic activity for gallium. Pharmacol. Rev. 1998, 50, 665-682. [PubMed]

17. Fernández-Cuenca, F.; Pascual, A.; Ribera, A.; Vila, J.; Bou, G.; Cisneros, J.M.; Rodríguez-Baño, J.; Pachón, J.; Martínez-Martínez, L.; Hospitalaria, G.d.E.d.I. Clonal diversity and antimicrobial susceptibility of Acinetobacter baumannii isolated in Spain. A nationwide multicenter study: GEIH-Ab project (2000). Enferm. Infecc. Microbiol. Clin. 2004, 22, 267-271. [CrossRef] 
18. Fernández-Cuenca, F.; Tomás-Carmona, M.; Caballero-Moyano, F.; Bou, G.; Martínez-Martínez, L.; Vila, J.; Pachón, J.; Cisneros, J.M.; Rodríguez-Baño, J.; Pascual, A.; et al. In vitro activity of 18 antimicrobial agents against clinical isolates of Acinetobacter spp.: Multicenter national study GEIH-REIPI-Ab 2010. Enferm. Infecc. Microbiol. Clin. 2013, 31, 4-9. [CrossRef] [PubMed]

19. García-Quintanilla, M.; Caro-Vega, J.M.; Pulido, M.R.; Moreno-Martínez, P.; Pachón, J.; McConnell, M.J. Inhibition of LpxC Increases Antibiotic Susceptibility in Acinetobacter baumannii. Antimicrob. Agents Chemother. 2016, 60, 5076-5079. [CrossRef]

20. Minandri, F.; Bonchi, C.; Frangipani, E.; Imperi, F.; Visca, P. Promises and failures of gallium as an antibacterial agent. Future Microbiol. 2014, 9, 379-397. [CrossRef] [PubMed] 